FACTA UNIVERSITATIS (NIŠ)

Ser. Math. Inform. Vol. 35, No 4 (2020), 899-917

https://doi.org/10.22190/FUMI2004899A

\title{
NEW INEQUALITIES OF OSTROWSKI TYPE FOR CO-ORDINATED CONVEX FUNCTIONS VIA GENERALIZED FRACTIONAL INTEGRALS *
}

\author{
Muhammad Aamir Ali, Hüseyin Budak and Zhiyue Zhang
}

(C) 2020 by University of Niš, Serbia | Creative Commons Licence: CC BY-NC-ND

Abstract. In this paper, we have established new inequalities of Ostrowski type for co-ordinated convex function by using generalized fractional integral. We have also discussed some special cases of our established results.

Keywords: inequalities of Ostrowski type; convex function; generalized fractional integral.

\section{Introduction}

In 1938, A. Ostowski established the following fascinating integral inequality [11].

Theorem 1.1. [11] Let $f:[a, b] \rightarrow \mathbb{R}$ be a differentiable function on $(a, b)$ whose derivative is bounded on $(a, b)$, i.e., $\left\|f^{\prime}(t)\right\|_{\infty}:=\sup \left|f^{\prime}(t)\right|<\infty$, for all $t \in(a, b)$. Then we have the following integral inequality:

$$
\left|f(x)-\frac{1}{b-a} \int_{a}^{b} f(x) d x\right| \leq\left[\frac{1}{4}+\frac{\left(x-\frac{a+b}{2}\right)}{(b-a)^{2}}\right](b-a)\left\|f^{\prime}\right\|_{\infty},
$$

for all $x \in[a, b]$. The $\frac{1}{4}$ is the best possible.

The inequality (1.1) can be rewritten in equivalent form as:

$$
\left|f(x)-\frac{1}{b-a} \int_{a}^{b} f(x) d x\right| \leq\left[\frac{(x-a)^{2}+(b-x)^{2}}{2(b-a)}\right]\left\|f^{\prime}\right\|_{\infty} .
$$

Received August 25, 2020; accepted October 07, 2020

2020 Mathematics Subject Classification. Primary 26D07, 26D10; Secondary 26D15, 26B15, 26 B25

* This project is partially supported by the National Natural Science Foundation of China(No. 11971241). 
Since 1938 when A. Ostrowski proved his famous inequality, (see, [11]), many mathematicians have been working about and around it, in many different directions and with a lot of applications in Numerical Analysis and Probability, etc.

Several generalizations of the Ostrowski integral inequality for mappings of bounded variation, Lipschitzian, monotonic, absolutely continuous, convex mappings and n-times differentiable mappings with error estimates for some special means and for some numerical quadrature rules have been considered by many authors. For recent results and generalizations concerning Ostrowski's inequality see [1]-[4], [6]-[15] and the references therein.

Let us consider now a bidimensional interval $\Delta:=[a, b] \times[c, d]$ in $\mathbb{R}^{2}$ with $a<b$ and $c<d$, a mapping $f: \Delta \rightarrow \mathbb{R}^{2}$ is said to be convex on $\Delta$ if the following inequality holds:

$f(t x+(1-t) z, t y+(1-t) w) \leq t f(x, y)+(1-t) f(z, w), \forall(x, y),(z, w) \in \Delta$ and $t \in[0,1]$.

The mapping $f$ is said to be concave on co-ordinates $\Delta$ if (1.3) holds in reversed direction.

A formal definition of co-ordinated convex (concave) functions may be expressed as:

Definition 1.1. [17]A function $f: \Delta \rightarrow \mathbb{R}$ is called co-ordinated convex on $\Delta$, for all $(x, u),(y, v) \in \Delta$ and $t, s \in[0,1]$, if it satisfies the following inequality:

$$
\begin{aligned}
& f(t x+(1-t) y, s u+(1-s) v) \\
\leq & t s f(x, u)+t(1-s) f(x, v)+s(1-t) f(y, u)+(1-t)(1-s) f(y, v) .
\end{aligned}
$$

The mapping $f$ is a co-ordinated concave on $\Delta$ if the inequality (1.4) holds in reversed direction for all $t, s \in[0,1]$ and $(x, u),(y, v) \in \Delta$.

In [5], Dragomir proved the following inequalities which is Hermite-Hadamard type inequalities for co-ordinated convex functions on the rectangle from the plane $\mathbb{R}^{2}$.

Theorem 1.2. Suppose that $f: \Delta \rightarrow \mathbb{R}$ is co-ordinated convex, then we have the 
following inequalities:

$$
\begin{aligned}
& f\left(\frac{a+b}{2}, \frac{c+d}{2}\right) \\
\leq & \frac{1}{2}\left[\frac{1}{b-a} \int_{a}^{b} f\left(x, \frac{c+d}{2}\right) d x+\frac{1}{d-c} \int_{c}^{d} f\left(\frac{a+b}{2}, y\right) d y\right] \\
\leq & \frac{1}{(b-a)(d-c)} \int_{a}^{b} \int_{c}^{d} f(x, y) d y d x \\
\leq & \frac{1}{4}\left[\frac{1}{b-a} \int_{a}^{b} f(x, c) d x+\frac{1}{b-a} \int_{a}^{b} f(x, d) d x\right. \\
& \left.+\frac{1}{d-c} \int_{c}^{d} f(a, y) d y+\frac{1}{d-c} \int_{c}^{d} f(b, y) d y\right] \\
\leq & \frac{f(a, c)+f(a, d)+f(b, c)+f(b, d)}{4} .
\end{aligned}
$$

The above inequalities are sharp. The inequalities in (1.5) holds in reversed direction if the mapping $f$ is a co-ordinated concave.

In [10], Latif et al. established following Ostrowski type inequalities for coordinated convex functions:

Theorem 1.3. Let $f: \Delta:=[a, b] \times[c, d] \rightarrow \mathbb{R}$ be twice partial differentiable mapping on $\Delta^{\circ}$ with $a<b, c<d, a, c \geq 0$ such that $\frac{\partial^{2} f}{\partial s \partial t} \in L(\Delta)$. If $\left|\frac{\partial^{2} f}{\partial s \partial t}\right|$ is convex on co-ordinates on $\Delta$ and $\left|\frac{\partial^{2} f}{\partial s \partial t}\right| \leq M,(x, y) \in \Delta$, then the following inequality holds:

$$
\begin{aligned}
& \left|f(x, y)+\frac{1}{(b-a)(d-c)} \int_{a}^{b} \int_{c}^{d} f(u, v) d v d u-A_{1}\right| \\
\leq & M\left[\frac{(x-a)^{2}+(b-x)^{2}}{2(b-a)}\right]\left[\frac{(y-c)^{2}+(d-y)^{2}}{2(d-c)}\right],
\end{aligned}
$$

where

$$
A_{1}=\frac{1}{d-c} \int_{c}^{d} f(x, v) d v+\frac{1}{b-a} \int_{c}^{d} f(u, y) d y
$$

Theorem 1.4. Let $f: \Delta:=[a, b] \times[c, d] \rightarrow \mathbb{R}$ be twice partial differentiable mapping on $\Delta^{\circ}$ with $a<b, c<d, a, c \geq 0$ such that $\frac{\partial^{2} f}{\partial s \partial t} \in L(\Delta)$. If $\left|\frac{\partial^{2} f}{\partial s \partial t}\right|^{q}$ is convex 
on co-ordinates on $\Delta, p, q>1, \frac{1}{p}+\frac{1}{q}=1$ and $\left|\frac{\partial^{2} f}{\partial s \partial t}(x, y)\right| \leq M,(x, y) \in \Delta$, then the following inequality holds:

$$
\begin{aligned}
& \left|f(x, y)+\frac{1}{(b-a)(d-c)} \int_{a}^{b} \int_{c}^{d} f(u, v) d v d u-A_{1}\right| \\
\leq & \frac{M}{(1+p)^{\frac{2}{p}}}\left[\frac{(x-a)^{2}+(b-x)^{2}}{2(b-a)}\right]\left[\frac{(y-c)^{2}+(d-y)^{2}}{2(d-c)}\right],
\end{aligned}
$$

where $A_{1}$ is defined in Theorem 1.3.

Theorem 1.5. Let $f: \Delta:=[a, b] \times[c, d] \rightarrow \mathbb{R}$ be twice partial differentiable mapping on $\Delta^{\circ}$ with $a<b, c<d, a, c \geq 0$ such that $\frac{\partial^{2} f}{\partial s \partial t} \in L(\Delta)$. If $\left|\frac{\partial^{2} f}{\partial s \partial t}\right|^{q}$ is convex on co-ordinates on $\Delta, q \geq 1$ and $\left|\frac{\partial^{2} f}{\partial s \partial t}(x, y)\right| \leq M,(x, y) \in \Delta$, then the following inequality holds:

$$
\begin{aligned}
& \left|f(x, y)+\frac{1}{(b-a)(d-c)} \int_{a}^{b} \int_{c}^{d} f(u, v) d v d u-A_{1}\right| \\
\leq & \frac{M}{4}\left[\frac{(x-a)^{2}+(b-x)^{2}}{2(b-a)}\right]\left[\frac{(y-c)^{2}+(d-y)^{2}}{2(d-c)}\right],
\end{aligned}
$$

where $A_{1}$ is defined in Theorem 1.3.

Theorem 1.6. Let $f: \Delta:=[a, b] \times[c, d] \rightarrow \mathbb{R}$ be twice partial differentiable mapping on $\Delta^{\circ}$ with $a<b, c<d, a, c \geq 0$ such that $\frac{\partial^{2} f}{\partial s \partial t} \in L(\Delta)$. If $\left|\frac{\partial^{2} f}{\partial s \partial t}\right|^{q}$ is concave on co-ordinates on $\Delta, p, q>1, \frac{1}{p}+\frac{1}{q}=1$, then the following inequality holds:

$$
\begin{aligned}
& \left|f(x, y)+\frac{1}{(b-a)(d-c)} \int_{a}^{b} \int_{c}^{d} f(u, v) d v d u-A_{1}\right| \\
\leq & \frac{1}{(1+p)^{\frac{2}{p}}(b-a)(d-c)}\left[( x - a ) ^ { 2 } \left\{(y-c)^{2}\left|\frac{\partial^{2}}{\partial s \partial t} f\left(\frac{x+a}{2}, \frac{y+c}{2}\right)\right|\right.\right. \\
& \left.+(d-y)^{2}\left|\frac{\partial^{2}}{\partial s \partial t} f\left(\frac{x+a}{2}, \frac{y+d}{2}\right)\right|\right\} \\
& +(b-x)^{2}\left\{(y-c)^{2}\left|\frac{\partial^{2}}{\partial s \partial t} f\left(\frac{x+b}{2}, \frac{y+c}{2}\right)\right|\right. \\
& \left.\left.+(d-y)^{2}\left|\frac{\partial^{2}}{\partial s \partial t} f\left(\frac{x+b}{2}, \frac{y+d}{2}\right)\right|\right\}\right],
\end{aligned}
$$

where $A_{1}$ is defined in Theorem 1.3.

In [9], Latif and Hussain established following Ostrowski type inequalities for co-ordinated convex function by using fractional integral: 
Theorem 1.7. Let $f: \Delta:=[a, b] \times[c, d] \rightarrow \mathbb{R}$ be twice partial differentiable mapping on $\Delta^{\circ}$ with $a<b, c<d, a, c \geq 0$ such that $\frac{\partial^{2} f}{\partial s \partial t} \in L(\Delta)$. If $\left|\frac{\partial^{2} f}{\partial s \partial t}\right|$ is convex on co-ordinates on $\Delta$ and $\left|\frac{\partial^{2} f}{\partial s \partial t}\right| \leq M,(x, y) \in \Delta$, then the following inequality holds for fractional integrals, with $\alpha, \beta>0$ :

$$
\begin{aligned}
& \left|\frac{\left[(x-a)^{\alpha}+(b-x)^{\alpha}\right]\left[(y-c)^{\beta}+(d-y)^{\beta}\right]}{(b-a)(d-c)} f(x, y)+A_{2}\right| \\
\leq & \frac{(\alpha \beta+2 \alpha+2 \beta+4)\left[(x-a)^{\alpha}+(b-x)^{\alpha}\right]\left[(y-c)^{\beta}+(d-y)^{\beta}\right]}{(b-a)(d-c)(\alpha+1)(\alpha+2)(\beta+1)(\beta+2)} M,
\end{aligned}
$$

where

$$
\begin{aligned}
A_{2}= & \frac{\Gamma(\alpha+1) \Gamma(\beta+a)}{(b-a)(d-c)}\left[J_{x-, y-}^{\alpha, \beta} f(a, c)+J_{x-, y+}^{\alpha, \beta} f(a, d)+J_{x+, y-}^{\alpha, \beta} f(b, c)\right. \\
& \left.+J_{x+, y+}^{\alpha, \beta} f(b, d)\right]-\frac{\left[(x-a)^{\alpha}+(b-x)^{\alpha}\right] \Gamma(\beta+1)}{(b-a)(d-c)}\left[J_{y-}^{\beta} f(x, c)+J_{y+}^{\beta} f(x, d)\right] \\
& -\frac{\left[(y-c)^{\beta}+(d-y)^{\beta}\right] \Gamma(\alpha+1)}{(b-a)(d-c)}\left[J_{x-}^{\alpha} f(a, y)+J_{x+}^{\alpha} f(b, y)\right] .
\end{aligned}
$$

Theorem 1.8. Let $f: \Delta:=[a, b] \times[c, d] \rightarrow \mathbb{R}$ be twice partial differentiable mapping on $\Delta^{\circ}$ with $a<b, c<d, a, c \geq 0$ such that $\frac{\partial^{2} f}{\partial s \partial t} \in L(\Delta)$. If $\left|\frac{\partial^{2} f}{\partial s \partial t}\right|^{q}$ is convex on co-ordinates on $\Delta, p, q>1, \frac{1}{p}+\frac{1}{q}=1$ and $\left|\frac{\partial^{2} f}{\partial s \partial t}(x, y)\right| \leq M,(x, y) \in \Delta$, then the following inequality holds for fractional integrals, with $\alpha, \beta>0$ :

$$
\begin{aligned}
& \left|\frac{\left[(x-a)^{\alpha}+(b-x)^{\alpha}\right]\left[(y-c)^{\beta}+(d-y)^{\beta}\right]}{(b-a)(d-c)} f(x, y)+A_{2}\right| \\
\leq & \frac{1}{(\alpha p+1)^{\frac{1}{p}}(\beta p+1)^{\frac{1}{p}}} \frac{\left[(x-a)^{\alpha}+(b-x)^{\alpha}\right]\left[(y-c)^{\beta}+(d-y)^{\beta}\right]}{(b-a)(d-c)} M,
\end{aligned}
$$

where $A_{2}$ is defined in Theorem 1.7.

Theorem 1.9. Let $f: \Delta:=[a, b] \times[c, d] \rightarrow \mathbb{R}$ be twice partial differentiable mapping on $\Delta^{\circ}$ with $a<b, c<d, a, c \geq 0$ such that $\frac{\partial^{2} f}{\partial s \partial t} \in L(\Delta)$. If $\left|\frac{\partial^{2} f}{\partial s \partial t}\right|^{q}$ is convex on co-ordinates on $\Delta, q \geq 1$ and $\left|\frac{\partial^{2} f}{\partial s \partial t}(x, y)\right| \leq M,(x, y) \in \Delta$, then the following inequality holds for fractional integrals, with $\alpha, \beta>0$ :

$$
\begin{aligned}
& \left|\frac{\left[(x-a)^{\alpha}+(b-x)^{\alpha}\right]\left[(y-c)^{\beta}+(d-y)^{\beta}\right]}{(b-a)(d-c)} f(x, y)+A_{2}\right| \\
\leq & \frac{1}{(\alpha p+1)^{\frac{1}{p}}(\beta p+1)^{\frac{1}{p}}} \frac{\left[(x-a)^{\alpha}+(b-x)^{\alpha}\right]\left[(y-c)^{\beta}+(d-y)^{\beta}\right]}{(b-a)(d-c)} M,
\end{aligned}
$$

where $A_{2}$ is defined in Theorem 1.7. 
Theorem 1.10. Let $f: \Delta:=[a, b] \times[c, d] \rightarrow \mathbb{R}$ be twice partial differentiable mapping on $\Delta^{\circ}$ with $a<b, c<d, a, c \geq 0$ such that $\frac{\partial^{2} f}{\partial s \partial t} \in L(\Delta)$. If $\left|\frac{\partial^{2} f}{\partial s \partial t}\right|^{q}$ is concave on co-ordinates on $\Delta, p, q>1, \frac{1}{p}+\frac{1}{q}=1$, then the following inequality holds for fractional integrals with $\alpha, \beta>0$ :

$$
\begin{aligned}
& \left|\frac{\left[(x-a)^{\alpha}+(b-x)^{\alpha}\right]\left[(y-c)^{\beta}+(d-y)^{\beta}\right]}{(b-a)(d-c)} f(x, y)+A_{2}\right| \\
\leq & \frac{1}{(1+\alpha p)^{\frac{1}{p}}(1+\beta p)^{\frac{1}{p}}(b-a)(d-c)} \\
& \times\left[( x - a ) ^ { \alpha + 1 } \left\{(y-c)^{\beta+1}\left|\frac{\partial^{2}}{\partial s \partial t} f\left(\frac{x+a}{2}, \frac{y+c}{2}\right)\right|\right.\right. \\
& +(d-y)^{\beta+1}\left|\frac{\partial^{2}}{\partial s \partial t} f\left(\frac{x+a}{2}, \frac{y+d}{2}\right)\right| \\
& +(b-x)^{\alpha+1}\left\{(y-c)^{\beta+1}\left|\frac{\partial^{2}}{\partial s \partial t} f\left(\frac{x+b}{2}, \frac{y+c}{2}\right)\right|\right. \\
& \left.\left.+(d-y)^{\beta+1}\left|\frac{\partial^{2}}{\partial s \partial t} f\left(\frac{x+b}{2}, \frac{y+d}{2}\right)\right|\right\}\right],
\end{aligned}
$$

where $A_{2}$ is defined in Theorem 1.7.

In [16], Sarikaya and Ertugral defined a new left-sided and right-sided generalized fractional integrals as follows:

$$
\begin{aligned}
& { }_{a+} I_{\varphi} f(x)=\frac{1}{\Gamma(\alpha)} \int_{a}^{x} \frac{\varphi(x-t)}{x-t} f(t) d t, \quad x>a \\
& { }_{b-} I_{\varphi} f(x)=\frac{1}{\Gamma(\alpha)} \int_{x}^{b} \frac{\varphi(t-x)}{t-x} f(t) d t, \quad x<b
\end{aligned}
$$

respectively, where $\varphi:[0, \infty) \rightarrow[0, \infty)$ a function which satisfies $\int_{0}^{1} \frac{\varphi(t)}{t} d t<\infty$.

In [17], Yildirim et al. defined generalized fractional integrals for two variable functions as follows:

Definition 1.2. $[17]$ Let $f \in L_{1}([a, b] \times[c, d])$. The generalized fractional integrals ${ }_{a+, c+} I_{\varphi, \psi},{ }_{a+, d-} I_{\varphi, \psi},{ }_{b-, c+} I_{\varphi, \psi}$ and ${ }_{b-, d-} I_{\varphi, \psi}$ are defined by

$$
{ }_{a+, c+} I_{\varphi, \psi} f(x, y)=\int_{a}^{x} \int_{c}^{y} \frac{\varphi(x-t)}{x-t} \frac{\psi(y-s)}{y-s} f(t, s) d s d t, \quad x>a, y>c,
$$




$$
\begin{aligned}
& { }_{a+, d-} I_{\varphi, \psi} f(x, y)=\int_{a}^{x} \int_{y}^{d} \frac{\varphi(x-t)}{x-t} \frac{\psi(s-y)}{s-y} f(t, s) d s d t, \quad x>a, y<d, \\
& { }_{b-, c+} I_{\varphi, \psi} f(x, y)=\int_{x}^{b} \int_{c}^{y} \frac{\varphi(t-x)}{t-x} \frac{\psi(y-s)}{y-s} f(t, s) d s d t, \quad x<b, y>c,
\end{aligned}
$$

and

$$
{ }_{b-, d-} I_{\varphi, \psi} f(x, y)=\int_{x}^{b} \int_{y}^{d} \frac{\varphi(t-x)}{t-x} \frac{\psi(s-y)}{s-y} f(t, s) d s d t, \quad x<b, y<d .
$$

Similar the above definitions, we can give the following integrals:

$$
\begin{aligned}
{ }_{a+} I_{\varphi} f(x, c) & =\int_{a}^{x} \frac{\varphi(x-t)}{x-t} f(t, c) d t, \quad x>a \\
{ }_{a+} I_{\varphi} f(x, d) & =\int_{a}^{x} \frac{\varphi(x-t)}{x-t} f(t, d) d t, \quad x>a, \\
{ }_{c+} I_{\psi} f(a, y) & =\int_{c}^{y} \frac{\psi(y-s)}{y-s} f(a, s) d s, \quad y>c,
\end{aligned}
$$

and

$$
{ }_{d-} I_{\psi} f(b, y)=\int_{y}^{d} \frac{\psi(s-y)}{s-y} f(b, s) d s, \quad y<d .
$$

The main objective of this paper is to establish new Ostrowski type inequalities for co-ordinated convex functions similar to $[9,10]$ by using generalized fractional integrals.

\section{Main Results}

Throughout this section, for clarity, we have defined

$$
\begin{array}{lll}
\Lambda_{1}(g) & =\int_{0}^{g} \frac{\varphi((x-a) t)}{t} d t, & \Lambda_{2}(g)=\int_{0}^{g} \frac{\varphi((b-x) t)}{t} d t \\
\Psi_{1}(h)=\int_{0}^{h} \frac{\psi((y-c) s)}{s} d s, & \Psi_{2}(h)=\int_{0}^{h} \frac{\psi((d-y) s)}{s} d s .
\end{array}
$$


Lemma 2.1. Let $f: \Delta:=[a, b] \times[c, d] \rightarrow \mathbb{R}$ be a twice differentiable mapping on $\Delta^{\circ}$ with $a<b, c<d$. If $\frac{\partial^{2} f}{\partial s \partial t} \in L(\Delta), a, c \geq 0$, then following identity holds for all $(x, y) \in \Delta$ :

$$
\text { 1) } \begin{aligned}
& \frac{\left[\Lambda_{2}(1)+\Lambda_{1}(1)\right]\left[\Psi_{2}(1)+\Psi_{1}(1)\right]}{(b-a)(d-c)} f(x, y)+A \\
= & \frac{(x-a)(y-c)}{(b-a)(d-c)} \int_{0}^{1} \int_{0}^{1} \Lambda_{1}(t) \Psi_{1}(s) \frac{\partial^{2}}{\partial s \partial t} f(t x+(1-t) a, s y+(1-s) c) d s d t \\
& -\frac{(x-a)(d-y)}{(b-a)(d-c)} \int_{0}^{1} \int_{0}^{1} \Lambda_{1}(t) \Psi_{2}(s) \frac{\partial^{2}}{\partial s \partial t} f(t x+(1-t) a, s y+(1-s) d) d s d t \\
& -\frac{(b-x)(y-c)}{(b-a)(d-c)} \int_{0}^{1} \int_{0}^{1} \Lambda_{2}(t) \Psi_{1}(s) \frac{\partial^{2}}{\partial s \partial t} f(t x+(1-t) b, s y+(1-s) c) d s d t \\
+ & \frac{(b-x)(d-y)}{(b-a)(d-c)} \int_{0}^{1} \int_{0}^{1} \Lambda_{2}(t) \Psi_{2}(s) \frac{\partial^{2}}{\partial s \partial t} f(t x+(1-t) b, s y+(1-s) d) d s d t
\end{aligned}
$$

where

$$
\begin{aligned}
A= & {\left[{ }_{x-, y-} I_{\varphi, \psi} f(a, c)+{ }_{x-, y+} I_{\varphi, \psi} f(a, d)+{ }_{x+, y-} I_{\varphi, \psi} f(b, c)+{ }_{x+, y+} I_{\varphi, \psi} f(b, d)\right] } \\
& -\Psi_{1}(1)\left[{ }_{x-} I_{\varphi} f(a, y)+{ }_{x+} I_{\varphi} f(b, y)\right]-\Psi_{2}(1)\left[{ }_{x-} I_{\varphi} f(a, y)+{ }_{x+} I_{\varphi} f(b, y)\right] \\
& -\Lambda_{1}(1)\left[{ }_{y-} I_{\psi} f(x, c)+{ }_{y+} I_{\psi} f(x, d)\right]-\Lambda_{2}(1)\left[{ }_{y-} I_{\psi} f(x, c)+{ }_{y+} I_{\psi} f(x, d)\right]
\end{aligned}
$$

Proof. Applying integration by parts and change of variables $u=t x+(1-t) a$ and $v=s y+(1-s) c$, we get

$$
\begin{aligned}
& \int_{0}^{1} \int_{0}^{1} \Lambda_{1}(t) \Psi_{1}(s) \frac{\partial^{2}}{\partial s \partial t} f(t x+(1-t) a, s y+(1-s) c) d s d t \\
= & \int_{0}^{1} \Lambda_{1}(t)\left\{\int_{0}^{1} \Psi_{1}(s) \frac{\partial^{2}}{\partial s \partial t} f(t x+(1-t) a, s y+(1-s) c) d s\right\} d t \\
= & \int_{0}^{1} \Lambda_{1}(t)\left\{\frac{\Psi_{1}(1)}{y-c} \frac{\partial}{\partial t} f(t x+(1-t) a, y)\right. \\
& \left.-\frac{1}{y-c} \int_{0}^{1} \frac{\psi((y-c) s)}{s} \frac{\partial}{\partial t} f(t x+(1-t) a, s y+(1-s) c) d s d t\right\} \\
= & \frac{\Psi_{1}(1)}{y-c} \int_{0}^{1} \Lambda_{1}(t) \frac{\partial}{\partial t} f(t x+(1-t) a, y) d t \\
& -\frac{1}{y-c} \int_{0}^{1} \frac{\psi((y-c) s)}{s}\left\{\int_{0}^{1} \Lambda_{1}(t) \frac{\partial}{\partial t} f(t x+(1-t) a, s y+(1-s) c) d t\right\} d s \\
= & \frac{\Psi_{1}(1)}{y-c}\left\{\frac{1}{x-a} \Lambda_{1}(1) f(x, y)-\frac{1}{x-a} \int_{0}^{1} \frac{\varphi((x-a) t)}{t} f(t x+(1-t) a, y) d t\right\} \\
& -\frac{1}{y-c} \int_{0}^{1} \frac{\psi((y-c) s)}{s}\left\{\frac{1}{x-a} \Lambda_{1}(1) f(x, s y+(1-s) c)\right.
\end{aligned}
$$




$$
\begin{aligned}
& \left.-\frac{1}{x-a} \int_{0}^{1} \frac{\varphi((x-a) t)}{t} f(t x+(1-t) a, s y+(1-s) c) d t\right\} d s \\
= & \frac{\Psi_{1}(1) \Lambda_{1}(1)}{(y-c)(x-a)} f(x, y)-\frac{\Psi_{1}(1)}{(x-a)(y-c)} \int_{0}^{1} \frac{\varphi((x-a) t)}{t} f(t x+(1-t) a, y) d t \\
& -\frac{\Lambda_{1}(1)}{(x-a)(y-c)} \int_{0}^{1} \frac{\psi((y-c) s)}{s} f(x, s y+(1-s) c) \\
& +\frac{1}{(x-a)(y-c)} \int_{0}^{1} \int_{0}^{1} \frac{\varphi((x-a) t)}{t} \frac{\psi((y-c) s)}{s} f(t x+(1-t) a, s y+(1-s) c) d s d t \\
= & \frac{\Psi_{1}(1) \Lambda_{1}(1)}{(y-c)(x-a)} f(x, y)-\frac{\Psi_{1}(1)}{(x-a)(y-c)}{ }_{x-} I_{\varphi} f(a, y) \\
& -\frac{\Lambda_{1}(1)}{(x-a)(y-c)} y-I_{\psi} f(x, c)+\frac{1}{(x-a)(y-c)}{ }_{x-, y-} I_{\varphi, \psi} f(a, c) .
\end{aligned}
$$

Similarly, applying the integration by parts, we also get

$$
\begin{aligned}
& \int_{0}^{1} \int_{0}^{1} \Lambda_{1}(t) \Psi_{2}(s) \frac{\partial^{2}}{\partial s \partial t} f(t x+(1-t) a, s y+(1-s) d) d s d t \\
= & -\frac{\Lambda_{1}(1) \Psi_{2}(1)}{(x-a)(d-y)} f(x, y)+\frac{\Psi_{2}(1)}{(x-a)(d-y)}{ }_{x-} I_{\varphi} f(a, y) \\
& +\frac{\Lambda_{1}(1)}{(x-a)(d-y)}{ }_{y+} I_{\psi} f(x, d)-\frac{1}{(x-a)(d-y)}{ }_{x-, y+} I_{\varphi, \psi} f(a, d), \\
= & -\frac{\Lambda_{2}(t) \Psi_{1}(s)}{(b-x)(y-c)} f(x, y)+\frac{\Psi_{1}(s)}{(b-x)(y-c)}{ }^{x+} I_{\varphi} f(b, y) \\
& +\frac{\Lambda_{2}(t)}{(b-x)(y-c)} y-I_{\psi} f(x, c)-\frac{1}{(b-x)(y-c)}{ }^{x+, y-} I_{\varphi, \psi} f(b, c), \\
= & \frac{\Lambda_{2}(t) \Psi_{2}(s)}{(b-x)(d-y)} f(x, y)-\frac{\Psi_{2}(s)}{(b-x)(d-y)}{ }^{x+} I_{\varphi} f(b, y) \\
& -\frac{\Lambda_{2}(t)}{(b-x)(d-y)} y+I_{\psi} f(x, d)-\frac{1}{(b-x)(y-c)}{ }^{1} \int^{1} \Lambda_{2}(t) \Psi_{2}(s) \frac{\partial^{2}}{\partial s \partial t} f(t x+(1-t) b, s y+(1-s) d) d s d t
\end{aligned}
$$

From (2.2)-(2.5) and dividing the resultant one by $(b-a)(d-c)$, we get our desired equality (2.1). 
Theorem 2.1. Let $f: \Delta:=[a, b] \times[c, d] \rightarrow \mathbb{R}$ be twice partial differentiable mapping on $\Delta^{\circ}$ with $a<b, c<d, a, c \geq 0$ such that $\frac{\partial^{2} f}{\partial s \partial t} \in L(\Delta)$. If $\left|\frac{\partial^{2} f}{\partial s \partial t}\right|$ is convex on co-ordinates on $\Delta$ and $\left|\frac{\partial^{2} f}{\partial s \partial t}\right| \leq M,(x, y) \in \Delta$, then the following inequality holds for generalized fractional integrals:

$$
\begin{aligned}
& \left|\frac{\left[\Lambda_{2}(1)+\Lambda_{1}(1)\right]\left[\Psi_{2}(1)+\Psi_{1}(1)\right]}{(b-a)(d-c)} f(x, y)+A\right| \\
\leq & \frac{M}{(b-a)(d-c)}\left[(x-a)(y-c) I_{1}+(x-a)(d-y) I_{2}\right. \\
& \left.+(b-x)(y-c) I_{3}+(b-x)(d-y) I_{4}\right],
\end{aligned}
$$

where

$$
\begin{array}{ll}
I_{1}=\int_{0}^{1} \int_{0}^{1} \Lambda_{1}(t) \Psi_{1}(s) d s d t, & I_{2}=\int_{0}^{1} \int_{0}^{1} \Lambda_{1}(t) \Psi_{2}(s) d s d t \\
I_{3}=\int_{0}^{1} \int_{0}^{1} \Lambda_{2}(t) \Psi_{1}(s) d s d t, & I_{4}=\int_{0}^{1} \int_{0}^{1} \Lambda_{2}(t) \Psi_{2}(s) d s d t
\end{array}
$$

and $A$ is defined in Lemma 2.1.

Proof. From Lemma 2.1, we get the following inequality that holds for all $(x, y) \in$ $\Delta:$

$$
\text { 2.7) } \begin{aligned}
\mid & \frac{\left[\Lambda_{2}(1)+\Lambda_{1}(1)\right]\left[\Psi_{2}(1)+\Psi_{1}(1)\right]}{(b-a)(d-c)} f(x, y)+A \mid \\
\leq & \frac{(x-a)(y-c)}{(b-a)(d-c)} \int_{0}^{1} \int_{0}^{1} \Lambda_{1}(t) \Psi_{1}(s)\left|\frac{\partial^{2}}{\partial s \partial t} f(t x+(1-t) a, s y+(1-s) c)\right| d s d t \\
& +\frac{(x-a)(d-y)}{(b-a)(d-c)} \int_{0}^{1} \int_{0}^{1} \Lambda_{1}(t) \Psi_{2}(s)\left|\frac{\partial^{2}}{\partial s \partial t} f(t x+(1-t) a, s y+(1-s) d)\right| d s d t \\
& +\frac{(b-x)(y-c)}{(b-a)(d-c)} \int_{0}^{1} \int_{0}^{1} \Lambda_{2}(t) \Psi_{1}(s)\left|\frac{\partial^{2}}{\partial s \partial t} f(t x+(1-t) b, s y+(1-s) c)\right| d s d t \\
& +\frac{(b-x)(d-y)}{(b-a)(d-c)} \int_{0}^{1} \int_{0}^{1} \Lambda_{2}(t) \Psi_{2}(s)\left|\frac{\partial^{2}}{\partial s \partial t} f(t x+(1-t) b, s y+(1-s) d)\right| d s d t .
\end{aligned}
$$

By the convexity of $\left|\frac{\partial^{2} f}{\partial s \partial t}\right|$ on co-ordinates on $\Delta$ and $\left|\frac{\partial^{2} f}{\partial s \partial t}\right| \leq M,(x, y) \in \Delta$, we 
have following inequalities:

$$
\begin{aligned}
& \int_{0}^{1} \int_{0}^{1} \Lambda_{1}(t) \Psi_{1}(s)\left|\frac{\partial^{2}}{\partial s \partial t} f(t x+(1-t) a, s y+(1-s) c)\right| d s d t \\
\leq & M \int_{0}^{1} \int_{0}^{1} t s \Lambda_{1}(t) \Psi_{1}(s) d s d t+M \int_{0}^{1} \int_{0}^{1} t(1-s) \Lambda_{1}(s) \Psi_{1}(s) d s d t \\
& +M \int_{0}^{1} \int_{0}^{1}(1-t) s \Lambda_{1}(t) \Psi_{1}(s) d t+M \int_{0}^{1} \int_{0}^{1}(1-t)(1-s) \Lambda_{1}(t) \Psi_{1}(s) d s d t \\
= & M \int_{0}^{1} \int_{0}^{1} \Lambda_{1}(t) \Psi_{1}(s) d s d t .
\end{aligned}
$$

Similarly, we have following inequalities

$$
\begin{aligned}
& \int_{0}^{1} \int_{0}^{1} \Lambda_{1}(t) \Psi_{2}(s)\left|\frac{\partial^{2}}{\partial s \partial t} f(t x+(1-t) a, s y+(1-s) d)\right| d s d t \\
& \leq M \int_{0}^{1} \int_{0}^{1} \Lambda_{1}(t) \Psi_{2}(s) d s d t \\
& \int_{0}^{1} \int_{0}^{1} \Lambda_{2}(t) \Psi_{1}(s)\left|\frac{\partial^{2}}{\partial s \partial t} f(t x+(1-t) b, s y+(1-s) c)\right| d s d t \\
& \leq M \int_{0}^{1} \int_{0}^{1} \Lambda_{2}(t) \Psi_{1}(s) d s d t \\
& \int_{0}^{1} \int_{0}^{1} \Lambda_{2}(t) \Psi_{2}(s)\left|\frac{\partial^{2}}{\partial s \partial t} f(t x+(1-t) b, s y+(1-s) d)\right| \\
& \leq M \int_{0}^{1} \int_{0}^{1} \Lambda_{2}(t) \Psi_{2}(s) d s d t .
\end{aligned}
$$

Now using (2.8)-(2.11) in (2.7), then we have our required inequality (2.6).

Remark 2.1. In Theorem 2.1, if we suppose $\varphi(t)=t$ and $\psi(s)=s$, then the inequality (2.6) becomes inequality (1.6).

Remark 2.2. In Theorem 2.1, if we take $\varphi(t)=\frac{t^{\alpha}}{\Gamma(\alpha)}$ and $\psi(s)=\frac{s^{\beta}}{\Gamma(\beta)}$, then the inequality (2.6) is reduced to the inequality (1.10).

Theorem 2.2. Let $f: \Delta:=[a, b] \times[c, d] \rightarrow \mathbb{R}$ be twice partial differentiable mapping on $\Delta^{\circ}$ with $a<b, c<d, a, c \geq 0$ such that $\frac{\partial^{2} f}{\partial s \partial t} \in L(\Delta)$. If $\left|\frac{\partial^{2} f}{\partial s \partial t}\right|^{q}$ is convex on co-ordinates on $\Delta, p, q>1, \frac{1}{p}+\frac{1}{q}=1$ and $\left|\frac{\partial^{2} f}{\partial s \partial t}(x, y)\right| \leq M,(x, y) \in \Delta$, then the following inequality holds for generalized fractional integrals:

$$
\begin{aligned}
& \left|\frac{\left[\Lambda_{2}(1)+\Lambda_{1}(1)\right]\left[\Psi_{2}(1)+\Psi_{1}(1)\right]}{(b-a)(d-c)} f(x, y)+A\right| \\
\leq & \frac{M}{(b-a)(d-c)}\left[(x-a)(y-c) J_{1}+(x-a)(d-y) J_{2}\right. \\
& \left.+(b-x)(y-c) J_{3}+(b-x)(d-y) J_{4}\right],
\end{aligned}
$$


where

$$
\begin{aligned}
& J_{1}=\left(\int_{0}^{1} \int_{0}^{1}\left(\Lambda_{1}(t) \Psi_{1}(s)\right)^{p} d s d t\right)^{\frac{1}{p}}, \quad J_{2}=\left(\int_{0}^{1} \int_{0}^{1}\left(\Lambda_{1}(t) \Psi_{2}(s)\right)^{p} d s d t\right)^{\frac{1}{p}} \\
& J_{3}=\left(\int_{0}^{1} \int_{0}^{1}\left(\Lambda_{2}(t) \Psi_{1}(s)\right)^{p} d s d t\right)^{\frac{1}{p}}, \quad J_{4}=\left(\int_{0}^{1} \int_{0}^{1}\left(\Lambda_{2}(t) \Psi_{2}(s)\right)^{p} d s d t\right)^{\frac{1}{p}}
\end{aligned}
$$

and $A$ is defined as in Lemma 2.1.

Proof. From Lemma 2.1 and the Hölder inequality, we have the following inequality that holds for all $(x, y) \in \Delta$ :

$$
\begin{aligned}
2.13) \mid & \left|\frac{\left.\Lambda_{2}(1)+\Lambda_{1}(1)\right]\left[\Psi_{2}(1)+\Psi_{1}(1)\right]}{(b-a)(d-c)} f(x, y)+A\right| \\
\leq & \frac{(x-a)(y-c)}{(b-a)(d-c)} \int_{0}^{1} \int_{0}^{1} \Lambda_{1}(t) \Psi_{1}(s)\left|\frac{\partial^{2}}{\partial s \partial t} f(t x+(1-t) a, s y+(1-s) c)\right| d s d t \\
& +\frac{(x-a)(d-y)}{(b-a)(d-c)} \int_{0}^{1} \int_{0}^{1} \Lambda_{1}(t) \Psi_{2}(s)\left|\frac{\partial^{2}}{\partial s \partial t} f(t x+(1-t) a, s y+(1-s) d)\right| d s d t \\
& +\frac{(b-x)(y-c)}{(b-a)(d-c)} \int_{0}^{1} \int_{0}^{1} \Lambda_{2}(t) \Psi_{1}(s)\left|\frac{\partial^{2}}{\partial s \partial t} f(t x+(1-t) b, s y+(1-s) c)\right| d s d t \\
& +\frac{(b-x)(d-y)}{(b-a)(d-c)} \int_{0}^{1} \int_{0}^{1} \Lambda_{2}(t) \Psi_{2}(s)\left|\frac{\partial^{2}}{\partial s \partial t} f(t x+(1-t) b, s y+(1-s) d)\right| d s d t \\
\leq & \frac{(x-a)(y-c)}{(b-a)(d-c)}\left(\int_{0}^{1} \int_{0}^{1}\left(\Lambda_{1}(t) \Psi_{1}(s)\right)^{p} d s d t\right)^{\frac{1}{p}} \\
& \times\left(\int_{0}^{1} \int_{0}^{1}\left|\frac{\partial^{2}}{\partial s \partial t} f(t x+(1-t) a, s y+(1-s) c)\right|^{q} d s d t\right)^{\frac{1}{q}} \\
& +\frac{(x-a)(d-y)}{(b-a)(d-c)}\left(\int_{0}^{1} \int_{0}^{1}\left(\Lambda_{1}(t) \Psi_{2}(s)\right)^{p} d s d t\right)^{\frac{1}{p}} \\
& \times\left(\int_{0}^{1} \int_{0}^{1}\left|\frac{\partial^{2}}{\partial s \partial t} f(t x+(1-t) a, s y+(1-s) d)\right|^{q} d s d t\right)^{\frac{1}{q}} \\
& +\frac{(b-x)(y-c)}{(b-a)(d-c)}\left(\int_{0}^{1} \int_{0}^{1}\left(\Lambda_{2}(t) \Psi_{1}(s)\right)^{p} d s d t\right)^{\frac{1}{p}} \\
& \times\left(\int_{0}^{1} \int_{0}^{1}\left|\frac{\partial^{2}}{\partial s \partial t} f(t x+(1-t) b, s y+(1-s) c)\right|^{q} d s d t\right)^{\frac{1}{q}} \\
& +\frac{(b-x)(d-y)}{(b-a)(d-c)}\left(\int_{0}^{1} \int_{0}^{1}\left(\Lambda_{2}(t) \Psi_{2}(s)\right)^{p} d s d t\right)^{\frac{1}{p}} \\
& \left(\int_{0}^{1}\left|\frac{\partial^{2}}{\partial s \partial t} f(t x+(1-t) b, s y+(1-s) d)\right|^{q} d s d t\right)^{\frac{1}{q}} . \\
&
\end{aligned}
$$


As we know that $\left|\frac{\partial^{2} f}{\partial s \partial t}(x, y)\right|^{q}$ is co-ordinated convex and $\left|\frac{\partial^{2} f}{\partial s \partial t}(x, y)\right|^{q} \leq M$, for all $(x, y) \in \Delta$, then we have the following inequality:

$$
\begin{aligned}
& \left(\int_{0}^{1} \int_{0}^{1}\left(\Lambda_{1}(t) \Psi_{1}(s)\right)^{p} d s d t\right)^{\frac{1}{p}} \\
& \times\left(\int_{0}^{1} \int_{0}^{1}\left|\frac{\partial^{2}}{\partial s \partial t} f(t x+(1-t) a, s y+(1-s) c)\right|^{q} d s d t\right)^{\frac{1}{q}} \\
\leq & M\left(\int_{0}^{1} \int_{0}^{1}\left(\Lambda_{1}(t) \Psi_{1}(s)\right)^{p} d s d t\right)^{\frac{1}{p}} .
\end{aligned}
$$

Analogously, we also have following inequalities

$$
\begin{aligned}
& \left(\int_{0}^{1} \int_{0}^{1}\left(\Lambda_{1}(t) \Psi_{2}(s)\right)^{p} d s d t\right)^{\frac{1}{p}} \\
\times & \left(\int_{0}^{1} \int_{0}^{1}\left|\frac{\partial^{2}}{\partial s \partial t} f(t x+(1-t) a, s y+(1-s) c d)\right|^{q} d s d t\right)^{\frac{1}{q}} \\
\leq & M\left(\int_{0}^{1} \int_{0}^{1}\left(\Lambda_{1}(t) \Psi_{2}(s)\right)^{p} d s d t\right)^{\frac{1}{p}}, \\
& \left(\int_{0}^{1} \int_{0}^{1}\left(\Lambda_{2}(t) \Psi_{1}(s)\right)^{p} d s d t\right)^{\frac{1}{p}} \\
& \times\left(\int_{0}^{1} \int_{0}^{1}\left|\frac{\partial^{2}}{\partial s \partial t} f(t x+(1-t) b, s y+(1-s) c)\right|^{q} d s d t\right)^{\frac{1}{q}} \\
\leq & M\left(\int_{0}^{1} \int_{0}^{1}\left(\Lambda_{2}(t) \Psi_{1}(s)\right)^{p} d s d t\right)^{\frac{1}{p}}, \\
& \left(\int_{0}^{1} \int_{0}^{1}\left(\Lambda_{2}(t) \Psi_{2}(s)\right)^{p} d s d t\right)^{\frac{1}{p}} \\
& \times\left(\int_{0}^{1} \int_{0}^{1}\left|\frac{\partial^{2}}{\partial s \partial t} f(t x+(1-t) b, s y+(1-s) d)\right|^{q} d s d t\right)^{\frac{1}{q}} \\
\leq & M\left(\int_{0}^{1} \int_{0}^{1}\left(\Lambda_{2}(t) \Psi_{2}(s)\right)^{p} d s d t\right)^{\frac{1}{p}} .
\end{aligned}
$$

By using (2.14)-(2.17) in (2.13), then we have our desired inequality (2.12).

Remark 2.3. In Theorem 2.2, if we suppose $\varphi(t)=t$ and $\psi(s)=s$, then the inequality (2.12) becomes inequality (1.7). 
Remark 2.4. In Theorem 2.2 , if we take $\varphi(t)=\frac{t^{\alpha}}{\Gamma(\alpha)}$ and $\psi(s)=\frac{s^{\beta}}{\Gamma(\beta)}$, then the inequality (2.12) is reduced to the inequality (1.11).

Theorem 2.3. Let $f: \Delta:=[a, b] \times[c, d] \rightarrow \mathbb{R}$ be twice partial differentiable mapping on $\Delta^{\circ}$ with $a<b, c<d, a, c \geq 0$ such that $\frac{\partial^{2} f}{\partial s \partial t} \in L(\Delta)$. If $\left|\frac{\partial^{2} f}{\partial s \partial t}\right|^{q}$ is convex on co-ordinates on $\Delta, q \geq 1$ and $\left|\frac{\partial^{2} f}{\partial s \partial t}(x, y)\right| \leq M,(x, y) \in \Delta$, then the following inequality holds for generalized fractional integrals:

$$
\begin{aligned}
& \left|\frac{\left[\Lambda_{2}(1)+\Lambda_{1}(1)\right]\left[\Psi_{2}(1)+\Psi_{1}(1)\right]}{(b-a)(d-c)} f(x, y)+A\right| \\
\leq & \frac{M}{(b-a)(d-c)}\left[(x-a)(y-c) I_{1}+(x-a)(d-y) I_{2}\right. \\
& \left.+(b-x)(y-c) I_{3}+(b-x)(d-y) I_{4}\right],
\end{aligned}
$$

where $I_{1}, I_{2}, I_{3}$ and $I_{4}$ are same as defined in Theorem 2.1 and $A$ is defined as in Lemma 2.1.

Proof. From Lemma 2.1 and the power mean inequality, we get the following inequality that holds for all $(x, y) \in \Delta$ :

$$
\begin{aligned}
2.19) \mid & \mid\left[\Lambda_{2}(1)+\Lambda_{1}(1)\right]\left[\Psi_{2}(1)+\Psi_{1}(1)\right] \\
\hline \leq-a)(d-c) & \frac{(x-a)(y-c)}{(b-a)(d-c)} \int_{0}^{1} \int_{0}^{1} \Lambda_{1}(t) \Psi_{1}(s)\left|\frac{\partial^{2}}{\partial s \partial t} f(t x+(1-t) a, s y+(1-s) c)\right| d s d t \\
& +\frac{(x-a)(d-y)}{(b-a)(d-c)} \int_{0}^{1} \int_{0}^{1} \Lambda_{1}(t) \Psi_{2}(s)\left|\frac{\partial^{2}}{\partial s \partial t} f(t x+(1-t) a, s y+(1-s) d)\right| d s d t \\
& +\frac{(b-x)(y-c)}{(b-a)(d-c)} \int_{0}^{1} \int_{0}^{1} \Lambda_{2}(t) \Psi_{1}(s)\left|\frac{\partial^{2}}{\partial s \partial t} f(t x+(1-t) b, s y+(1-s) c)\right| d s d t \\
& +\frac{(b-x)(d-y)}{(b-a)(d-c)} \int_{0}^{1} \int_{0}^{1} \Lambda_{2}(t) \Psi_{2}(s)\left|\frac{\partial^{2}}{\partial s \partial t} f(t x+(1-t) b, s y+(1-s) d)\right| d s d t . \\
\leq & \frac{(x-a)(y-c)}{(b-a)(d-c)}\left(\int_{0}^{1} \int_{0}^{1} \Lambda_{1}(t) \Psi_{1}(s) d s d t\right) \\
& \times\left(\int_{0}^{1} \int_{0}^{1} \Lambda_{1}(t) \Psi_{1}(s)\left|\frac{\partial^{2}}{\partial s \partial t} f(t x+(1-t) a, s y+(1-s) c)\right|^{q} d s d t\right)^{\frac{1}{q}} \\
& +\frac{(x-a)(d-y)}{(b-a)(d-c)}\left(\int_{0}^{1} \int_{0}^{1} \Lambda_{1}(t) \Psi_{2}(s) d s d t\right)^{1-\frac{1}{q}} \\
& \times\left(\int_{0}^{1} \int_{0}^{1} \Lambda_{1}(t) \Psi_{2}(s)\left|\frac{\partial^{2}}{\partial s \partial t} f(t x+(1-t) a, s y+(1-s) d)\right|^{q} d s d t\right)^{\frac{1}{q}}
\end{aligned}
$$




$$
\begin{aligned}
& +\frac{(b-x)(y-c)}{(b-a)(d-c)}\left(\int_{0}^{1} \int_{0}^{1} \Lambda_{2}(t) \Psi_{1}(s) d s d t\right)^{1-\frac{1}{q}} \\
& \times\left(\int_{0}^{1} \int_{0}^{1} \Lambda_{2}(t) \Psi_{1}(s)\left|\frac{\partial^{2}}{\partial s \partial t} f(t x+(1-t) b, s y+(1-s) c)\right|^{q} d s d t\right)^{\frac{1}{q}} \\
& +\frac{(b-x)(d-y)}{(b-a)(d-c)}\left(\int_{0}^{1} \int_{0}^{1} \Lambda_{2}(t) \Psi_{2}(s) d s d t\right)^{1-\frac{1}{q}} \\
& \times\left(\int_{0}^{1} \int_{0}^{1} \Lambda_{2}(t) \Psi_{2}(s)\left|\frac{\partial^{2}}{\partial s \partial t} f(t x+(1-t) b, s y+(1-s) d)\right|^{q} d s d t\right)^{\frac{1}{q}}
\end{aligned}
$$

As we know that $\left|\frac{\partial^{2} f}{\partial s \partial t}(x, y)\right|^{q}$ is co-ordinated convex and $\left|\frac{\partial^{2} f}{\partial s \partial t}(x, y)\right| \leq M$, for all $(x, y) \in \Delta$, then we have the following inequality:

$$
\begin{aligned}
& \int_{0}^{1} \int_{0}^{1} \Lambda_{1}(t) \Psi_{1}(s)\left|\frac{\partial^{2}}{\partial s \partial t} f(t x+(1-t) a, s y+(1-s) c)\right|^{q} d s d t \\
& \leq M^{q} \int_{0}^{1} \int_{0}^{1} \Lambda_{1}(t) \Psi_{1}(s) .
\end{aligned}
$$

Similarly, we have following inequalities:

$$
\begin{aligned}
& \int_{0}^{1} \int_{0}^{1} \Lambda_{1}(t) \Psi_{2}(s)\left|\frac{\partial^{2}}{\partial s \partial t} f(t x+(1-t) a, s y+(1-s) d)\right|^{q} d s d t \\
& \leq M^{q} \int_{0}^{1} \int_{0}^{1} \Lambda_{1}(t) \Psi_{2}(s) \\
& \int_{0}^{1} \int_{0}^{1} \Lambda_{2}(t) \Psi_{1}(s)\left|\frac{\partial^{2}}{\partial s \partial t} f(t x+(1-t) b, s y+(1-s) c)\right|^{q} d s d t \\
& \leq M^{q} \int_{0}^{1} \int_{0}^{1} \Lambda_{2}(t) \Psi_{1}(s) \\
& \int_{0}^{1} \int_{0}^{1} \Lambda_{2}(t) \Psi_{2}(s)\left|\frac{\partial^{2}}{\partial s \partial t} f(t x+(1-t) b, s y+(1-s) d)\right|^{q} d s d t \\
& \leq M^{q} \int_{0}^{1} \int_{0}^{1} \Lambda_{2}(t) \Psi_{2}(s) .
\end{aligned}
$$

By using (2.20)-(2.23) in (2.19), we have our desired inequality (2.18).

Remark 2.5. In Theorem 2.3, if we suppose $\varphi(t)=t$ and $\psi(s)=s$, then the inequality (2.18) becomes inequality (1.8). 
Remark 2.6. In Theorem 2.3, if we take $\varphi(t)=\frac{t^{\alpha}}{\Gamma(\alpha)}$ and $\psi(s)=\frac{s^{\beta}}{\Gamma(\beta)}$, then the inequality (2.18) reduces to the inequality (1.12).

Theorem 2.4. Let $f: \Delta:=[a, b] \times[c, d] \rightarrow \mathbb{R}$ be twice partial differentiable mapping on $\Delta^{\circ}$ with $a<b, c<d, a, c \geq 0$ such that $\frac{\partial^{2} f}{\partial s \partial t} \in L(\Delta)$. If $\left|\frac{\partial^{2} f}{\partial s \partial t}\right|^{q}$ is concave on co-ordinates on $\Delta, p, q>1, \frac{1}{p}+\frac{1}{q}=1$, then the following inequality hold for generalized fractional integrals:

$$
\begin{aligned}
& \left|\frac{\left[\Lambda_{2}(1)+\Lambda_{1}(1)\right]\left[\Psi_{2}(1)+\Psi_{1}(1)\right]}{(b-a)(d-c)} f(x, y)+A\right| \\
\leq & \frac{1}{(b-a)(d-c)}\left[(x-a)(y-c)\left|\frac{\partial^{2}}{\partial s \partial t} f\left(\frac{x+a}{2}, \frac{y+c}{2}\right)\right| J_{1}\right. \\
& +(x-a)(d-y)\left|\frac{\partial^{2}}{\partial s \partial t} f\left(\frac{x+a}{2}, \frac{y+d}{2}\right)\right| J_{2} \\
& +(b-x)(y-c)\left|\frac{\partial^{2}}{\partial s \partial t} f\left(\frac{x+b}{2}, \frac{y+c}{2}\right)\right| J_{3} \\
& \left.+(b-x)(d-y)\left|\frac{\partial^{2}}{\partial s \partial t} f\left(\frac{x+b}{2}, \frac{y+d}{2}\right)\right| J_{4}\right],
\end{aligned}
$$

where $J_{1}, J_{2}, J_{3}$ and $J_{4}$ are same as defined in Theorem 2.2.

Proof. From Lemma 2.1 and the Hölder inequality, we have the following inequality that holds for all $(x, y) \in \Delta$ :

$$
\begin{aligned}
(2.25) \mid & \left|\frac{\left.\Lambda_{2}(1)+\Lambda_{1}(1)\right]\left[\Psi_{2}(1)+\Psi_{1}(1)\right]}{(b-a)(d-c)} f(x, y)+A\right| \\
\leq & \frac{(x-a)(y-c)}{(b-a)(d-c)} \int_{0}^{1} \int_{0}^{1} \Lambda_{1}(t) \Psi_{1}(s)\left|\frac{\partial^{2}}{\partial s \partial t} f(t x+(1-t) a, s y+(1-s) c)\right| d s d t \\
& +\frac{(x-a)(d-y)}{(b-a)(d-c)} \int_{0}^{1} \int_{0}^{1} \Lambda_{1}(t) \Psi_{2}(s)\left|\frac{\partial^{2}}{\partial s \partial t} f(t x+(1-t) a, s y+(1-s) d)\right| d s d t \\
& +\frac{(b-x)(y-c)}{(b-a)(d-c)} \int_{0}^{1} \int_{0}^{1} \Lambda_{2}(t) \Psi_{1}(s)\left|\frac{\partial^{2}}{\partial s \partial t} f(t x+(1-t) b, s y+(1-s) c)\right| d s d t \\
& +\frac{(b-x)(d-y)}{(b-a)(d-c)} \int_{0}^{1} \int_{0}^{1} \Lambda_{2}(t) \Psi_{2}(s)\left|\frac{\partial^{2}}{\partial s \partial t} f(t x+(1-t) b, s y+(1-s) d)\right| d s d t \\
\leq & \frac{(x-a)(y-c)}{(b-a)(d-c)}\left(\int_{0}^{1} \int_{0}^{1}\left(\Lambda_{1}(t) \Psi_{1}(s)\right)^{p} d s d t\right)^{\frac{1}{p}} \\
& \times\left(\int_{0}^{1} \int_{0}^{1}\left|\frac{\partial^{2}}{\partial s \partial t} f(t x+(1-t) a, s y+(1-s) c)\right|^{q} d s d t\right)^{\frac{1}{q}}
\end{aligned}
$$




$$
\begin{aligned}
& +\frac{(x-a)(d-y)}{(b-a)(d-c)}\left(\int_{0}^{1} \int_{0}^{1}\left(\Lambda_{1}(t) \Psi_{2}(s)\right)^{p} d s d t\right)^{\frac{1}{p}} \\
& \times\left(\int_{0}^{1} \int_{0}^{1}\left|\frac{\partial^{2}}{\partial s \partial t} f(t x+(1-t) a, s y+(1-s) d)\right|^{q} d s d t\right)^{\frac{1}{q}} \\
& +\frac{(b-x)(y-c)}{(b-a)(d-c)}\left(\int_{0}^{1} \int_{0}^{1}\left(\Lambda_{2}(t) \Psi_{1}(s)\right)^{p} d s d t\right)^{\frac{1}{p}} \\
& \times\left(\int_{0}^{1} \int_{0}^{1}\left|\frac{\partial^{2}}{\partial s \partial t} f(t x+(1-t) b, s y+(1-s) c)\right|^{q} d s d t\right)^{\frac{1}{q}} \\
& +\frac{(b-x)(d-y)}{(b-a)(d-c)}\left(\int_{0}^{1} \int_{0}^{1}\left(\Lambda_{2}(t) \Psi_{2}(s)\right)^{p} d s d t\right)^{\frac{1}{p}} \\
& \times\left(\int_{0}^{1} \int_{0}^{1}\left|\frac{\partial^{2}}{\partial s \partial t} f(t x+(1-t) b, s y+(1-s) d)\right|^{q} d s d t\right)^{\frac{1}{q}} .
\end{aligned}
$$

Since $\left|\frac{\partial^{2} f}{\partial s \partial t}\right|$ is concave on co-ordinates on $\Delta$, so an application of (1.5) with inequalities in reversed direction, we have following inequalities:

$$
\begin{aligned}
& \int_{0}^{1} \int_{0}^{1}\left|\frac{\partial^{2}}{\partial s \partial t} f(t x+(1-t) a, s y+(1-s) c)\right|^{q} d s d t \\
& \leq\left|\frac{\partial^{2}}{\partial s \partial t} f\left(\frac{x+a}{2}, \frac{y+c}{2}\right)\right|^{q}, \\
& \int_{0}^{1} \int_{0}^{1}\left|\frac{\partial^{2}}{\partial s \partial t} f(t x+(1-t) a, s y+(1-s) d)\right|^{q} d s d t \\
& \leq\left|\frac{\partial^{2}}{\partial s \partial t} f\left(\frac{x+a}{2}, \frac{y+d}{2}\right)\right|^{q}, \\
& \int_{0}^{1} \int_{0}^{1}\left|\frac{\partial^{2}}{\partial s \partial t} f(t x+(1-t) b, s y+(1-s) c)\right|^{q} d s d t \\
& \leq\left|\frac{\partial^{2}}{\partial s \partial t} f\left(\frac{x+b}{2}, \frac{y+c}{2}\right)\right|^{q}, \\
& \leq\left|\frac{\partial^{2}}{\partial s \partial t} f\left(\frac{x+b}{2}, \frac{y+d}{2}\right)\right|^{q} \cdot \\
& \int_{0}^{1} \int_{0}^{1}\left|\frac{\partial^{2}}{\partial s \partial t} f(t x+(1-t) b, s y+(1-s) d)\right|^{q} d s d t \\
& \leq
\end{aligned}
$$

By using (2.26)-(2.29) in (2.25), then we have our desired inequality (2.24). 
Remark 2.7. In Theorem 2.4, if we suppose $\varphi(t)=t$ and $\psi(s)=s$, then the inequality (2.24) becomes inequality (1.9).

Remark 2.8. In Theorem 2.4, if we take $\varphi(t)=\frac{t^{\alpha}}{\Gamma(\alpha)}$ and $\psi(s)=\frac{s^{\beta}}{\Gamma(\beta)}$, then the inequality (2.24) is reduced to the inequality (1.13).

\section{R E F E R E N C E S}

1. M. Alomari, M. Darus, S.S. Dragomir and P. Cerone, Ostrowski type inequalities for functions whose derivatives are s-convex in the second sense, Applied Mathematics Letters Volume 23, Issue 9, September 2010, Pages 1071-1076.

2. M. Alomari and M. Darus, Some Ostrowskiís type inequalities for convex functions with applications, RGMIA, 13(1) (2010), Article 3. [ONLINE: http://a jmaa.org/RGMIA/v13n1.php].

3. N. S. Barnett and S. S. Dragomir, An Ostrowski type inequality for double integrals and applications for cubature formulae, Soochow J. Math., 27(1), (2001), 109-114.

4. P. Cerone and S.S. Dragomir, Ostrowski type inequalities for functions whose derivatives satisfy certain convexity assumptions, Demonstratio Math., 37 (2004), no. 2, 299-308.

5. S.S. Dragomir, On Hadamards inequality for convex functions on the co-ordinates in a rectangle from the plane. Taiwan. J. Math. 4, 775-788 (2001).

6. S.S. Dragomir and A. Sofo, Ostrowski type inequalities for functions whose derivatives are convex, Proceedings of the 4th International Conference on Modelling and Simulation, November 11-13, 2002. Victoria University, Melbourne, Australia. RGMIA Res. Rep. Coll., 5 (2002), Supplement, Article 30. [ONLINE: http://rgmia.vu.edu.au/v5(E).html]

7. S. S. Dragomir, N. S. Barnett and P. Cerone, An n-dimensional version of Ostrowskiís inequality for mappings of Hölder type, RGMIA Res. Pep. Coll., 2(2), (1999), 169-180.

8. S.S. Dragomir and C.E.M. Pearce, Selected Topics on Hermite-Hadamard Inequalities and Applications, RGMIA Monographs, Victoria University, 2000. Online [http://www.staff.vu.edu.au/RGMIA/monographs/hermite_hadamard.html].

9. M. A. Latif, S. Hussain, New inequalities of Ostowski type for co-ordinated convex functions via fractional integrals, J. Fract. Calc. Appl, 2(2012), no. 9, 1-15.

10. M. A. Latif, S. Hussain and S. S. Dragomir, New Ostrowski type inequalities for co-ordinated convex functions, RGMIA Research Report Collection, 14(2011), Article 49. [ONLINE:http://www.a jmaa.org/RGMIA/v14.php].

11. A. M. Ostrowski, Über die absolutabweichung einer differentiebaren funktion von ihrem integralmitelwert, Comment. Math. Helv. 10(1938), 226-227.

12. B. G. Pachpatte, On an inequality of Ostrowski type in three independent variables, J. Math. Anal. Appl., 249(2000), 583-591. 
13. B. G. Pachpatte, On a new Ostrowski type inequality in two independent variables, Tamkang J. Math., 32(1), (2001), 45-49

14. B. G. Pachpatte, A new Ostrowski type inequality for double integrals, Soochow J. Math., 32(2), (2006), 317-322.

15. M. Z. Sarikaya, On the Ostrowski type integral inequality, Acta Math. Univ. Comenianae, Vol. LXXIX, 1(2010), pp. 129-134.

16. M.Z. Sarikaya and F. Ertuğral, On the generalized Hermite-Hadamard inequalities, Annals of the University of Craiova - Mathematics and Computer Science Series, in press.

17. M. E. Yildirim, M. Z. Sarikaya, H. BUDAK and H. Yildirim, Some Hermite-Hadamard type integral inequalities for co-ordinated convex functions via generalized fractional integrals, December 2017, https://www.researchgate.net/publication/321803898.

Muhammad Aamir Ali

Jiangsu Key Laboratory of NSLSCS

School of Mathematical Sciences

Nanjing Normal University

210023, Nanjing, China

mahr.muhammad . aamir@gmail.com

Hüseyin Budak

aculty of Science and Arts

Department of Mathematics

Düzce University

Düzce, Turkey

hsyn. budak@gmail . com

Zhiyue Zhang

Jiangsu Key Laboratory of NSLSCS

School of Mathematical Sciences

Nanjing Normal University

210023, Nanjing, China

05298@njnu.edu.cn 